\title{
ESTUDIO DE INOCUIDAD DE Salmonella enterica, SUBESPECIE enterica, SEROTIPO ENTERITIDIS, VAR. DANYSZ, LISINA NEGATIVA EN POLLOS PARRILLEROS
}

\author{
Natalia Rodríguez A. ${ }^{1}$, Eliana Icochea D’A. ${ }^{2}$, Sonia Calle E. ${ }^{3}$ y Norma Noé M.
}

\section{Abstract}

The Salmonella enterica, serotipe Enteritidis, var Danysz, negative lysine is used as a component in a product for biological control of rodents. The present study evaluated the innocuosness of the strain in 120 1-day old Cobb Vantress broiler chicks of both sexes. They were divided into 3 groups: Group A was fed at 5, 6 and 7 days of age with a commercial broiler feed containing $20 \%$ of the rat poison, Group B received $1 \mathrm{ml}$ containing $10^{8} \mathrm{CFU}$ of the bacteria in the crop at 8 days of age, and Group $\mathrm{C}$ was kept as a control group. Clinical signs, mortality, recovery of the experimental strain used from liver and spleen, body weight, and serological response were recorded. All birds were weighed every week. Two chicks were ramdomly selected and necropsied from each group on daily basis since the fifth day and for a week. Serum samples and selected tissues were collected for bacteriological culture. Additionally, serum samples were collected at day 21, 28, and 35 after challenge. The bacteriological and serological analysis were negative for Salmonella sp.; however, one bird on day 28 and 2 birds on day 35 after challenge from group A were positive in the agglutination test toSalmonella sp. In one of them, a Salmonella sp. positive lysine was isolated. Body weight differences among groups were not found by the seventh week.

Key words: Salmonella, rodents, lysine, broilers

\section{Resumen}

Se realizó un estudio con la cepa Salmonella enterica, subespecie enterica, serotipo Enteritidis, var. Danysz, lisina negativa, principio activo de un producto comercial usado para el control biológico de roedores, a fin de evaluar su inocuidad en pollos parrilleros. Se usaron 120 aves de 1 día de edad de ambos sexos de la línea Cobb Vantress divididas en 3 grupos. El grupo A recibió un alimento comercial que contenía $20 \%$ del producto raticida al $5^{\circ}, 6^{\circ}$ y $7^{\circ}$ día de edad. El grupo $\mathrm{B}$ fue inoculado en el buche al $8^{\circ}$ día de edad con $10^{8}$ UFC de la bacteria $(1 \mathrm{ml})$ y el grupo C permaneció como control. Se registraron signos clínicos, mortalidad, peso corporal individual por semana, respuesta serológica y la recuperación de la cepa experimental de Salmonella en hígado y bazo. A partir del

\footnotetext{
${ }^{1}$ Práctica privada. E-mail: nataliarodriguezayala@hotmail.com

${ }^{2}$ Laboratorio de Patología Aviar, FMV-UNMSM

${ }^{3}$ Laboratorio de Microbiología y Parasitología Veterinaria, FMV-UNMSM

${ }^{4}$ Laboratorio de Medicina Veterinaria Preventiva, FMV-UNMSM
} 
quinto día del desafío y durante una semana, dos aves por grupo fueron necropsiadas. Adicionalmente, se tomaron muestras de sangre a todas las aves a los 21, 28 y 35 días post desafío y en las aves sacrificadas, para la detección de anticuerpos contra Salmonella mediante la prueba de aglutinación en placa. No se encontraron lesiones macroscópicas compatibles con infección por Salmonella. Los resultados del estudio bacteriológico y serológico fueron negativos a Salmonella sp; sin embargo, un ave a los 28 días y 2 aves a los 35 días post desafío (3/18) del grupo A dieron positivo a la prueba de aglutinación para Salmonella sp. De estas, en una se aisló Salmonella sp. tipificada como lisina positiva. Los pesos corporales a la sétima semana no mostraron diferencias significativas entre grupos.

Palabras clave: Salmonella, roedores, lisina, broiler

\section{INTRODUCCIÓN}

La salmonelosis en humanos es una de las enfermedades de transmisión alimentaria de gran importancia en salud pública. La gastroenteritis causada por Salmonella en el hombre ha sido usualmente asociada al consumo de productos avícolas (Parimal et al., 2001). Desde el punto de vista de la salud pública, las diferencias de virulencia entre cepas de Salmonella enteritidis en pollos es de menor relevancia que las diferencias en su habilidad de propagación en el lote y en su capacidad de contaminar los huevos de las aves infectadas (Gast y Benson, 1996). La S. enteritidis ocasiona una disminución de la ganancia de peso en pollos jóvenes y afecta la uniformidad de los lotes, resultando en considerables pérdidas económicas para los productores (Dhillon et al., 1999).

La Samonella se encuentra en el agua, en el medio ambiente y en el alimento, por lo que los huevos de las reproductoras pueden ser contaminados a partir de diversas fuentes. El huevo, dependiendo del serotipo, la agresividad y la adaptación de la Salmonella al huésped, puede tener cuatro rutas de contaminación: ovarios, oviducto, cloaca y medio ambiente (Baxter-Jones, 1996). La diseminación horizontal en la parvada puede ocurrir también a partir de diversas fuentes (Hamilton, 2003). Los pollitos recién nacidos son altamente susceptibles a la infección por Salmonella enteritidis (Gast y Holt, 1999).
La importancia de los roedores como diseminadores y reservorios de Salmonella es conocida (Henzler y Opitz, 1992). Se ha encontrado una relación entre presencia de roedores y galpones de ponedoras con medio ambiente positivos a Salmonella, y donde, además, se observa una alta prevalencia de $S$. enterica, serotipo Enteritidis, en ratones de estos galpones (Garber et al., 2003).

Los roedores son transmisores de enfermedades que afectan al hombre y a los animales, como la peste bubónica, salmonelosis, brucelosis, leptospirosis, listeriosis, encefalitis y otras que llegan a través de sus mordeduras, orina, heces, $\mathrm{y}$ de las pulgas que los parasitan (Collazos y Castro, 1997). En la industria avícola, los roedores, además, consumen y deterioran (rotura de sacos y alimento contaminado con orina) una importante cantidad de alimento destinado a las aves. Se estima que el alimento que consumen por día equivale al $10 \%$ de su peso corporal. Además, ocasionan daños al material de empaque, destrozan las bandejas para huevos, se comen los huevos en los galpones y en bodegas de almacenamiento, atacan a las aves, matan a los pollitos y deterioran las instalaciones (Díaz, 1999).

En el control de roedores se emplean principalmente raticidas químicos, tanto de acción rápida como de acción lenta o anticoagulantes. Los primeros presentan la desventaja de causar un síndrome de rechazo por parte de los roedores, ya que apren- 
den a asociar muerte con consumo (Collazos y Castro, 1997). Los segundos requieren mayor tiempo de exposición y debido a que su antídoto específico es la vitamina $\mathrm{K}$, la cual se encuentra en la ración de las aves, se presentan problemas de ineficacia.

En algunos países, incluyendo el Perú, se viene usando el control biológico a través de la bacteria Salmonella enterica, sub especie enterica, serovar Enteritidis, var. Danysz, según la nomenclatura más reciente (Popoff, 2001). La cepa fue caracterizada por el Centro Colaborador de la OMS para Enteropatógenos, el Servicio de Laboratorios de Salud Pública en Colindale, Londres (PHLS, por las siglas en inglés), clasificándose como Salmonella enteritidis, e indicándose que la característica bioquímica más notoria, y que la diferencia de la mayoría de cepas de Salmonella sp., es ser lisina negativa (Threlfall, 1996).

Se considera que este método es más eficaz que el químico, porque produce una epizootia que puede causar la eliminación de la población de roedores sin que los pollos de carne se vean afectados con una infección clínica o subclínica por esta bacteria; sin embargo, hay otros riesgos para las aves que deben ser investigados.

\section{Materiales y Métodos}

\section{Lugar de estudio}

La fase de campo se realizó en las instalaciones de la unidad experimental del Laboratorio de Patología Aviar y el estudio bacteriológico se realizó en el Laboratorio de Microbiología de la Facultad de Medicina Veterinaria de la Universidad Nacional Mayor de San Marcos.

\section{Animales y tamaño de muestra}

En el cálculo del tamaño muestral se consideró información de experimentos pre- vios donde se evaluó el peso corporal de aves inoculadas experimentalmente con Salmonella sp. y Salmonella enteritidis en diferentes rangos (13-19\%). El número de aves necesario para el experimento, según el "Tamaño de Muestra de Comparación de Medias", fue de 15 como mínimo. Sin embargo, para el estudio se utilizaron 120 pollos de carne de la línea Cobb Vantress de ambos sexos de un día de edad, que fueron distribuidos en 3 grupos. Se sacrificaron 12 aves en el momento de la recepción para la evaluación bacteriológica y serológica con la finalidad de comprobar su estado libre de Salmonella. Se les suministró agua potable y alimento $\mathrm{ad}$ limitum, usándose un alimento comercial para pollos de carne.

\section{Diseño experimental}

Se usaron grupos de 36 aves cada uno. El grupo A fue alimentado con el producto raticida (Biorat $\left.{ }^{\circledR}\right)$ para control biológico $(20 \%$ de la ración) en el $5^{\circ}, 6^{\circ}$ y $7^{\circ}$ día de edad, el grupo $\mathrm{B}$ fue inoculado directamente en el buche al $8^{\circ}$ día de edad con un cultivo de la bacteria Salmonella enterica, subespecie enterica, serotipo Enteritidis, var. Danysz, lisina negativa ( $1 \mathrm{ml} \mathrm{con} 10^{8}$ UFC) y el grupo $\mathrm{C}$ fue el control negativo.

El periodo experimental fue de 7 semanas, donde se registraron signos clínicos y la mortalidad. Además, se registró semanalmente el peso de los pollitos.

\section{Evaluación patológica y serológica}

Se sacrificaron 2 aves/día/grupo al azar por 7 días y a partir del $5^{\circ}$ día post inoculación (13 días de edad) o del término de la suplementación del raticida (12 días de edad). Las aves del grupo control se sacrificaron a partir de los 12 días de edad. Además, se sacrificaron 2 aves por grupo a los 35 días de edad. Se registraron los cambios patológicos macroscópicos en las necropsias. 
El estudio bacteriológico se realizó mediante cultivo indirecto (en caldo de enriquecimiento) y cultivo directo (en agar) de muestras de hígado y bazo, y a través de pruebas bioquímicas en las colonias resultantes. Se utilizaron los medios LIA, KIA, prueba de producción de $\mathrm{H}_{2} \mathrm{~S}$, prueba de motilidad o SIM, urea y la prueba de API 20.

Se tomaron muestras de sangre a las aves sacrificadas y a la totalidad de aves los días 21, 31 y 40 post inoculación o al término de la suplementación. Los sueros se analizaron mediante la prueba de aglutinación rápida en placa para Salmonella sp. (Antígeno Nobilis ${ }^{\circledR}$ Antigen Intervet).

\section{Análisis estadísticos}

Se evaluó la mortalidad, peso corporal, signos clínicos, lesiones y el porcentaje de recuperación de Salmonella a partir de muestras de hígado y bazo, así como la respuesta serológica a la prueba de aglutinación en placa.

La mortalidad y el peso corporal se correlacionó con los demás parámetros mencionados. El peso corporal se analizó mediante ANOVA y la prueba de diferencias de medias de Duncan.

\section{Resultados y Discusión}

Los sueros iniciales y el estudio bacteriológico del ciego y del saco vitelino al primer día de edad fueron negativos a anticuerpos contra Salmonella sp. y la presencia de Salmonella, respectivamente. Asimismo, los resultados de los exámenes del alimento consumido por las aves indicaron que se encontraba libre de contaminación con Salmonella sp. Sin embargo, en una prueba se encontró una cepa con imagen similar a Salmonella, que con la prueba API 20 se logró identificar como Citrobacter freundi.
Ninguna de las aves de los grupos tratados ni del grupo control mostraron depresión, diarrea, disminución en el consumo de alimento o agua de bebida ni ningún otro signo clínico de enfermedad, que son característicos de las afecciones producidas por Salmonella sp. (Dhillon et al., 1998). No obstante, se tuvo una mortalidad acumulada del $4.4 \%$, cifra esperada en la crianza de pollos parrilleros en batería. Las causas de mortalidad fueron colibacilosis y estrés de calor. No se encontraron lesiones compatibles con infección por Salmonella enteritidis en las aves sacrificadas (Gorham et al., 1994).

La evaluación serológica se hizo para determinar la capacidad del raticida en producir seroconversión positiva en las aves. Sin embargo, todas las muestras de los grupos B y $\mathrm{C}$ fueron negativas a anticuerpos contra Salmonella sp., con excepción de una muestra colectada en el día 28 y 2 muestras del día 35, ambas del grupo A, que hacen un total de 3/18 aves positivas a la prueba de aglutinación de placa para Salmonella sp.

Las aves serológicamente positivas no se vieron afectadas en el peso corporal, ni manifestaron signos clínicos o mostraron lesiones a la necropsia. Se presumió que la seroconversión de las tres aves se debió a la infección por la cepa de Salmonella usada en el experimento. En el examen bacteriológico se logró aislar una bacteria en una de las tres aves, y que mediante la prueba bioquímica se demostró que era Salmonella sp. lisina positiva.

Los análisis bacteriológicos realizados a partir de las muestras de hígado y bazo colectadas en las aves sacrificadas con el fin de recuperar la cepa de desafío fueron negativos, indicando que la Salmonella enterica lisina negativa no produce una diseminación sistémica, aun cuando el periodo de colección de muestras para el presente trabajo fue en la etapa de mayor probabilidad de recuperación bacteriana $\left(5^{\circ}\right.$ a $11^{\circ}$ día post inoculación), tal como fue obtenido por otros investi- 
gadores, pero usando para el desafío una cepa de Salmonella lisina positiva (Gast y Benson, 1996; Gast y Holt, 1999; Parimal et al., 2001; Dhillon et al., 1999) .

En base a la respuesta de la cepa de Salmonella enteritidis con relación a la utilización de la lisina, se puede inferir que la Salmonella aislada fue diferente a la utilizada en el experimento. La $S$. enteritidis es una bacteria ampliamente distribuida, con múltiples hospederos y puede llegar a una parvada por transmisión vertical y horizontal, por contaminación de otros animales como aves silvestres, roedores e insectos, alimento contaminado y polvo (Gast, 2003). Debido a que el experimento no fue realizado en unidades de estricto aislamiento, los roedores o aves silvestres podrían haber contaminando el alimento o el agua a bajos niveles llegando a afectar a uno de los pollos.

Existen reportes que demuestran una disminución del peso corporal (13-19\%) en las aves por infecciones con Salmonella sp. aunque no desarrollan la enfermedad ni se presentan casos de mortalidad (Dhillon et al., 1998; Parimal et al., 2001). Sin embargo, en el presente experimento no se afectaron los parámetros productivos de las aves desafiadas con la cepa en estudio.

\section{Conclusiones}

- La ausencia de signos clínicos, lesiones y mortalidad en las aves inoculadas o alimentadas con Salmonella enterica, sub especie enterica, serotipo enteritidis, var. Danysz, lisina negativa, evidencian que este germen no es patogénico, y por lo tanto se le puede considerar como inocua para las aves.

- La ocurrencia de serorreacción de algunas aves contra esta bacteria no permite concluir que el raticida que contiene esta bacteria no ocasiona infecciones subclínicas.

\section{Literatura Citada}

1. Baxter-Jones, C. 1996. Control de la transmisión vertical de Salmonella. Avicultura Profesional 14: 18-19.

2. Collazos, R; J. Castro. 1997. Los roedores dañinos: algunos aspectos del control químico y bacteriológico. Rev. Inv. Pec. IVITA 8: 1-9.

3. Dhillon, A.S.; B. Alisantosa; H.L. Shivaprasad; O. Jack; D. Schaberg; D. Bandli. 1999. Pathogenicity of Salmonella enteritidis phage types 4, 8 and 23 in broiler chicks. Avian Dis. 43: 506-515.

4. Dhillon, A.S.; O.K. Jack; H.L. Shivaprasad; D.M. Schaberg; D.V. Bandli. 1998. Experimental infection of Salmonella enteritidis in broilers chicks. Proc. 50 $0^{\text {th }}$ Western Poultry Disease Conference. Davis, California, USA.

5. Díaz, F.J. 1999. Bioseguridad en el control de plagas. En: Bioseguridad en la industria avícola. p 115-130. Ed. Quebecor Impreandes. Santa Fé de Bogotá, Colombia.

6. Garber, L.; M. Smeltzer; P. FedorkaCray; S. Ladely; K. Ferris. 2003. Salmonella enterica serotipe Enteritidis in table egg layer house enviroments and in mice in U.S. layer houses and associated risk factors. Avian Dis. 47: 134-142.

7. Gast, R.K. 2003. Paratyphoid infections. Diseases of Poultry. $11^{\text {th }}$ ed. p 583-599. Iowa State Press. USA.

8. Gast, R.K.; P.S. Holt. 1999. Experimental horizontal transmission of Salmonella enteritidis strains (Phage types 4, 8 and 13A) in chicks. Avian Dis. 43: 774-778.

9. Gast, R.K.; S.T. Benson. 1996. Intestinal colonization and organ invasion in chicks experimentally infected with Salmonella enteritidis phage type 4 and other phage types isolated from poultry in the United States. Avian Dis. 40: 853857. 
10. Gorham, S.L.; K. Kadavil; E. Vaughan; H. Lambert; J. Abel; B. Pert. 1994. Gross and microscopic lesions in young chickens experimentally infected with Salmonella enteritidis. Avian Dis. 38: 816-821.

11. Hamilton, E. 2003. Salmonella enteritidis y Salmonella typhimurium. Poultry Industry Council, Canada. Disponible en: http://www.poultryindustry council.ca/Factsheets/Factsheets/ fact105.htm

12. Henzler, D.J.; H.M. Opitz. 1992. The role of mice in the epizootiology of Salmonella enteritidis infection on chicken layer chicks. Avian Dis. 36: 625-631.
13. Parimal, R.; A.S. Dhillon; H.L. Shivaprasad; D.M. Shaberg; D. Bandli; S. Johnson. 2001. Pathogenicity of different sero-groups of avian salmonellas in SPF chickens. Avian Dis. 45: 922-937.

14. Popoff, M.Y. 2001. Antigenic formulas of the Salmonella serovars. $8^{\text {th }} \mathrm{ed.} \mathrm{WHO}$ Collaborating Centre for Reference and Research on Salmonella, Institut Pasteur. Paris France. 6 p.

15. Threlfall, E.J. 1996. Report on work carried out on Salmonella enteritidis var. Issatschenko. Laboratory of Enteric Pathogens, Central Public Health Laboratory. London, UK. 